\title{
Content and Context Aware Strategies for QoS Support in VANETs
}

\author{
Gianluca Rizzo*, Maria Rita Palattella ${ }^{\dagger}$, Torsten Braun ${ }^{\ddagger}$, and Thomas Engel ${ }^{\dagger}$ \\ *HES SO Valais, Switzerland, gianluca.rizzo@hevs.ch \\ ${ }^{\dagger}$ SnT, University of Luxembourg (Luxembourg), \{maria-rita.palattella, thomas.engel\}@uni.lu \\ ${ }^{\ddagger}$ University of Bern, Switzerland, braun@inf.unibe.ch
}

\begin{abstract}
The increasing interest in autonomous coordinated driving and in proactive safety services, exploiting the wealth of sensing and computing resources which are gradually permeating the urban and vehicular environments, is making provisioning of high levels of QoS in vehicular networks an urgent issue. At the same time, the spreading model of a smart car, with a wealth of infotainment applications, calls for architectures for vehicular communications capable of supporting traffic with a diverse set of performance requirements. So far efforts focused on enabling a single specific QoS level. But the issues of how to support traffic with tight QoS requirements (no packet loss, and delays inferior to $1 \mathrm{~ms}$ ), and of designing a system capable at the same time of efficiently sustaining such traffic together with traffic from infotainment applications, are still open. In this paper we present the approach taken by the CONTACT project to tackle these issues. The goal of the project is to investigate how a VANET architecture, which integrates contentcentric networking, software-defined networking, and context aware floating content schemes, can properly support the very diverse set of applications and services currently envisioned for the vehicular environment.
\end{abstract}

Index Terms-Vehicular Ad-hoc Networks, Quality-of-Service, Content-Centric Networking, Software-Defined Networking, Floating Content

\section{INTRODUCTION}

The recent years have seen an increasing interest in autonomous coordinated driving and in proactive road safety services, fueled by the need to increase the efficiency of resource (energy, infrastructure) utilization in transportation, and by the need to decrease car accident rates. The increasing level of penetration of sensing, computing and communicating devices (from sensor rich connected vehicles to smartphones, to smart objects) offers new opportunities for an increased level of automation (driving, coordination between vehicles, traffic management) and new mechanisms for preventing hazards on the road.

In order to satisfy the communication needs of such applications, Vehicular Ad Hoc Networks (VANETs) [1] have emerged, supporting data exchange between vehicles and/or between vehicle and fixed infrastructure. They are typically characterized by a dynamic topology, by intermittent, often short lived wireless connectivity, and they are usually based on a cooperative, decentralized communication paradigm.

VANETs hold the potential to enable a wide range of applications and services [2]. So far the focus has been mainly on safety applications, such as road awareness, accident/intersection warning, traffic vigilance, which can have a huge impact in avoiding traffic accidents and increasing safety of road transportation [3].

Recently, autonomous and coordinated driving applications are emerging as a strong driver for the adoption of VANETs [4] [5]. An automated-driving system relies extensively on the vehicle's local sensors. The intrinsic limitations of these sensors often prevent planning and maneuvers that would require information from farther distances and/or occluded spaces. The use of short-range, low latency wireless communication among vehicles are then a method to mitigate this issue and expand the reach of the sensory information locally available at each vehicle [6].

At the same time, initiatives such as the Apple Car suggest that an increasingly important role is going to be played by infotainment vehicular applications. Relieving drivers from the need to focus on what happens on the road will also contribute to this trend, as the demand for in vehicle connectivity and entertainment services will likely rise as a consequence.

All these applications are however characterized by very different communication requirements. The majority of the state of the art on VANETs aimed primarily at efficiently supporting delay-tolerant and best-effort applications, such as infotainment and traffic congestion notifications, easier to support in such a challenging communication environment [2]. Moreover, existing approaches focus on a single application or a family of applications, proposing ad-hoc solutions, which can hardly be extended to a multi-service scenario [7]-[9]. Safety critical services, such as cooperative collision avoidance, are highly impacted by network performance and by the variability of the vehicular environment, as shown in [7]. A MAC scheme capable of delivering emergency messages with low delays and no packet loss was proposed in [8]. However, such approach applies to scenarios where transmission of high priority messages is rare and messages are short. Carspeak [9] proposes a content centric network mechanism for decreasing collisions, in single hop scenario. Although the approach is promising, the solution is ad hoc for hidden obstacle detection, and it is unclear how to extend it to other V2V communication scenarios, as well as how to support traffic from other applications.

To date, how to design a VANET capable of reliably satisfying the stringent requirements of safety and autonomous driving applications while at the same time flexibly and adaptively supporting infotainment traffic, remains an open issue. More- 
over, it is quite well understood that the use of context is a powerful instrument for improving the efficiency of communications in VANETs. Nonetheless, solutions proposed so far, while giving an idea of the potential performance gains achievable, are specific for a given application and context, and it is not clear how to extend them to other applications and scenarios.

From the review of related work, it is clear that no single technical approach can yield a solution that is able to support all communication requirements of current and future applications. The variety of communication requirements and the challenges of a highly volatile communication environment call for a diversified approach. We believe that the enabling of multiple applications with diversified QoS requirements should come from tackling the problem at several layers of the communication architecture, and by adapting different mechanisms. Moreover, many of the most promising available results emphasize the usefulness of context information in optimizing vehicular communications.

In this paper, we present the approach which is at the basis of the CONTACT (CONtext and conTent Aware CommunicaTions for QoS support in VANETs) project. The main goal of the project is to design an architecture for the provision of various levels of Quality of Service (QoS) in VANETs. This will be achieved through the investigation of several communication strategies capable of adapting, at the same time, to the highly volatile and unstable vehicular environment, to content attributes and properties, and to a diversified set of application performance requirements.

To this aim, CONTACT will investigate and exploit the use of three different emerging approaches: Content-Centric Networking (CCN) [10], Software Defined Networking (SDN) [11], and Floating Content (FC) [12], [13]. Even though not designed for VANETs, they can bring big advantages to vehicular applications, by improving content availability, replication and distribution.

$\mathrm{CCN}$ introduces (content) name-based addressing instead of host-based addressing. This can be beneficial for communications in highly mobile network scenarios such as vehicular networks, where host addresses are not very meaningful. Interest messages with content names can be used for content discovery. SDN, with its centralized view of network resources, may help in setting up data flows between discovered content sources and requesters, handling efficiently dynamic (re)allocation of resources/channels, and distribution of content (e.g., by reducing amount of geobroadcast messages). Finally, CCN as well as FC techniques could be used to improve content availability for delay tolerant communications.

The rest of this paper is organized as follows. In Section II we describe in detail the CONTACT approach, and how each single technique (CCN, SDN, FC) can be applied in VANETs. In Section III we present some preliminary ideas on how to integrate the three different approaches within the CONTACT architecture. Finally, in Section IV we draw some concluding remarks.

\section{A Multipronged Approach to QOS PROVISION IN VANETS}

In this section we describe the multipronged, cross-layer approach adopted in the CONTACT project, which builds on three different emerging paradigms: Content-Centric Networking, Software Defined Networking, and Floating Content. We describe how each paradigm can be exploited, individually, to enhance QoS support in VANETs.

\section{A. CCN design for VANETs}

A content centric network $(\mathrm{CCN})$ delivers content objects efficiently by focusing on the needs of the user rather than on the location of the content, which changes continuously in a vehicular network. Users request content using a content name instead of an IP address [10]. Nodes receiving the request (Interest packet) check whether they have a piece of content with that data name, and send it back to the requesting node. CCN routers can cache content and disseminate it efficiently. Thus, CCN is attractive in VANETs as it potentially enables more efficient utilization of network resources and helps reducing network traffic. CCNx [14] provides an open source reference implementation of CCN. CCN is also well suited for continuous media flows [15]. Source mobility can be supported by a routing-based approach, indirection, or resolution [16]. However, these approaches have drawbacks in terms of convergence and scalability.

Early works investigated the applicability of existing MANET routing protocols for mobile $\mathrm{CCN}$ based on analytical models [17]. A hierarchical CCN routing scheme based on distributed meta information has been implemented in [18]. Listen First, Broadcast Later (LFBL) [19] limits forwarding of Interests at every node based on its relative distance to the content source. These works assume continuous network connectivity and do not consider intermittent connectivity.

Previous research [20], [21] has demonstrated that CCN works well in wireless networks. Interests can be broadcasted and nodes hosting the requested content can directly respond [19], [22], [23]. Overheard cached copies can satisfy requests from other nodes, but broadcast requests can trigger transmissions from multiple content sources resulting in collisions and duplicate content transmissions. Thus, broadcast must be used carefully in wireless networks. We proposed a strategy called Dynamic Unicast [24] to reduce collisions and the time to retrieve content, but, such strategy does not support multi-hop communications, as required in VANETs.

1) CCN routing for multi-hop dynamic VANETs: CCN can efficiently support information exchange in highly mobile environments as it is the case in VANETs. Its main advantage is represented by the integration of content and node discovery, which is important in case of rather short contact times and short lived paths between two peers. With the CONTACT project, we foresee to apply CCN in VANETs by developing mechanisms for routing and forwarding $\mathrm{CCN}$ Interest and data messages across multi-hop VANETs. Such mechanisms will be based on the previously developed Dynamic Unicast scheme [24], originally designed for single-hop scenario.The 
CONTACT approach aims to extend Dynamic Unicast by support for multi-hop communications. For this, we propose

- step 1: to disseminate the first Interest by (controlled) flooding or by $\mathrm{CCN}$-based forwarding if entries for the content in the Forwarding Information Base (FIB) can be found;

- step 2: to return the first Data message using multi-hop routing on the reverse path from the content source to the requester;

- step 3: similarly to Dynamic Unicast, subsequent communication will then be point-to-point between requester and content source. For this phase, we need a routing protocol that can handle high mobility of vehicles. Our previous research on routing protocols for highly mobile scenarios resulted in the development of opportunistic routing protocols based on geographic forwarding [25]. Such geographic routing mechanism for point-to-point communication between requester and identified content source is promising for VANETs.

2) Mobility Support: Mobility of content sources can be supported by permanent piggybacking the latest geographic position of a source node on the Data messages from content source to the requester. If a path between requester and content source breaks, several options can be considered.

- The requester can again start a new content search by disseminating an Interest using flooding or $\mathrm{CCN}$-based forwarding as in step 1 above.

- The node that detected the path break can repair the path using the geographic routing protocol.

- The node that detected the path break can initiate a new content search, e.g., by using the same mechanisms as the requester in step 1 or by sending the Interest into an area, where the content source is assumed to be.

3) Quality of Service Support: $\mathrm{CCN}$ can be used for any application such as audio/video streaming or conferencing. In those cases, it might be useful to provide QoS support, in particular in wireless networks with limited network bandwidth. With the CONTACT project, we aim to design and implement QoS support for the geographic routing approach proposed for $\mathrm{CCN}$-based content exchange. We propose to estimate the required bandwidth and other resources for a Data stream based on tracking Interests' round trip time estimations. Each Interest message forwarded towards a content source creates an entry in the Pending Interest Table of a $\mathrm{CCN}$ router. A router thus knows that Data has been requested and that Data messages will arrive on the reverse path. By measuring the round trip time between itself and a content source, the amount of required network bandwidth resources can be estimated and resources can be allocated and reserved. In case of lacking resources, appropriate shaping actions for outgoing Interest messages have to be initiated.

\section{B. SDN design for VANETs}

Software-Defined Networking (SDN), emerged as a new dynamic, manageable, cost-effective, and adaptable architecture, ideal for the high-bandwidth, and dynamic nature of today's applications [11]. SDN separates data (forwarding) plane from control plane, enhancing the programmability of the network by external applications. In other words, a SDN controller decides, according to the requirements of applications, how traffic flows will be forwarded within the network while network devices (switches, routers) simply forward the packets, following the per-flow rules installed by the controller. SDN was initially designed with the intention of overcoming the drawbacks of traditional networks, such as manual configuration, and further maintenance of every single device in the network, latency in path-recovery due to distributed approach, etc. By applying SDN, network management and (re)configuration, resource allocation, network troubleshooting and debugging can become easier.

SDN was initially designed for wired networks, such as data centers. Being a dynamic and adaptable paradigm, rather than a rigid architecture, it is feasible to apply it in a different context from the one it was initially designed for. Recently, SDN has been recognized as an attractive and promising approach for wireless and mobile networks too [26], VANETs included, as detailed hereafter.

1) SDN architecture for VANETs: In $2014 \mathrm{Ku}$ et al. proposed the first SDN architecture for VANETs [27], and they highlighted the benefits offered by the SDN paradigm, such as path selection for rerouting traffic and avoiding congestion, channel selection and allocation for guaranteeing low-latency to safety applications, among others.

Several factors have to be considered when designing a SDN-based architecture for VANETs: 1) the level of control owned by the SDN controller (full, or distributed with other SDN entities localized in road side units (RSUs), and base stations); 2) the medium used for data and control communication (WiFi, or infrastructure-based: 3G, LTE). In a fog-based SDN architecture for VANETs proposed in literature [28], the control of the network is shared between the SDN controller and local agents, which are located both within some RSUs and wireless nodes. Vehicles are considered as SDN wireless nodes, equipped with WiMax/3G/4G/ LTE interfaces mostly for control communication, and WiFi/WAVE interface for data communication.

2) Resource management support: Only $75 \mathrm{MHz}$ and 50 $\mathrm{MHz}$ of the bandwidth in the $5 \mathrm{GHz}$ spectrum have been allocated for Dedicated Short Range Communications (DSRC) in VANETs, both in the United States and in Europe. In the reserved bandwidth a single Control Channel $(\mathrm{CCH})$ and several Service Channels (SCHs) exist. By default, control and safety messages are broadcast on the $\mathrm{CCH}$, while other kinds of DSRC messages are transmitted on SCHs. The limited number of available channels calls for efficient channel selection/resource allocation methods. A SDN controller having full knowledge of the network status (i.e. context) is a good candidate for selecting and allocating channels to different classes of traffic (i.e. content-based). A first contribution in this area has been provided by Liu et al. [29], who have designed a cooperative data dissemination scheme for VANETs. An SDN controller, localized within the RSU delivers schedul- 
ing decisions to vehicles, instructing them which channel to tune to, and which data to transmit/receive. Moreover, SDN can dynamically adapt to topology changes, and reconfigure resource allocations to provide the expected QoS/QoE. For instance, if a vehicle $\mathrm{A}$ is out of the coverage range of the RSU, but it can get the content service from another neighbor vehicle $\mathrm{B}$, which is currently within the RSUs coverage range, the SDN controller could allocate more resources to vehicle $\mathrm{B}$ to allow content delivery to vehicle $\mathrm{A}$ in a $\mathrm{CCN}$ fashion.

3) Content delivery support: In future VANETs vehicles will produce and exchange a large amount of content for enabling new collaborative services such as autonomous driving. At the same time they will be using infotainment services, and getting bandwidth demanding content. Due to the (different) type and amount of content, new approaches for improving content distribution in VANETs are needed. We will investigate within CONTACT how an SDN controller can implement policy based methods for reducing replication (broadcasting), without affecting content availability (reliability). The potential of SDN to improve the management of content delivery has been already highlighted in related work. A Type-Based Content Distribution (TBCD) method has been proposed [30] for improving content caching and forwarding in a SDN-enabled VANET. TBCD adopts a push-and-pull approach for delivering content, based on the type of content, and on the number of users interested in it. SDN has also the potential to improve and reduce the amount of broadcast messages exchanged in VANETs, with the resulting advantage of efficiently using network resources, reducing collision. An SDN architecture for GeoBroadcast in VANETs has been recently defined in [31]. With CONTACT, we plan to further investigate similar techniques.

\section{FC design for VANETs}

Floating Content (FC) [12], [13] is an opportunistic communication scheme to support infrastructure-less distributed content sharing over a given geographic area, called Anchor Zone (AZ). The objective of FC is to ensure the availability of some content items within the $\mathrm{AZ}$ for a given time period by replicating them opportunistically to users, who come in contact within the AZ, so that the content items can float within the AZ. Existing works on FC performance focus on determining the conditions under which a content item floats with high probability [13] and on application-level performance modeling [32].

Floating Content has been proposed in VANETs for accident warnings, traffic jam notifications, and for all event-based messaging of local scope and of relatively short temporal interest [33], [34]. Indeed, the ephemeral nature of such messages, coupled with the necessity of optimizing storage and communications, call for ways to efficiently control replication and diffusion of floating messages. FC suits particularly such context, in which fixed infrastructure for sensing/detecting a problem (accident, traffic jam) and for spreading a warning might be missing or inadequate. It is also considered for safety messages generated by cars (e.g. medical conditions relative to the driver), for which $\mathrm{V} 2 \mathrm{~V}$ communications are the natural choice, as they naturally incorporate context. In [33] authors study the storage capabilities of floating information for the case of vehicular ad-hoc networks. The performance of floating content-like service on a highway as well as in a city setting has been evaluated in [34]. For such cases, floating content expands the reach of a message beyond the transmission radius of the originator, allowing the reception of the message (and therefore take actions, such as a detour and/or adaptation of speed and inter vehicle distance) well before reaching the location of the event. In addition, the opportunistic replication of the message allows incrementing the efficiency with which it is dispatched, decreasing the impact of interference, collisions, etc.

Being conceived for settings where users are mobile, FC adapts well to volatile and dynamic environments without support from fixed infrastructure. The main challenge in FC indeed is to build services with an acceptable level of (application level) performance on top of an unreliable, best effort communication service such as one based on opportunistic replication. However, FC has so far been mainly conceived for smartphone users, and hence assessed in settings where users move at walking speed. Ad hoc vehicular communications, however, push the possibilities of FC to the limit. As speeds are higher, interaction times are lower making it challenging to recognize neighbors, set up connections and exchange data. Moreover, the typical spatio-temporal patterns of users are radically different than those of walking mobile users. Also the type of applications and associated communication requirements differ significantly.

All these works show the necessity to adapt the FC paradigm, on one side, to vehicular network environment, and on the other, to the specific requirements of the service/application supported.

1) FC modeling and optimization: One of the critical aspects of the FC paradigm is tuning its main service parameters (e.g. shape, size and speed of the anchor zone and of the replication zone) to satisfy application requirements. Indeed, several versions of the FC paradigm have already been proposed, each with its own definition of success rate, its shape and relationship between the aforementioned parameters [12], [32]. Some related works [32] show that performance of floating content is strictly related to the communication requirements of the specific service, and of the target performance at the application level.

In order to take this aspect into account, it is necessary to first identify the main families of vehicular services which could be supported by FC, grouping application with similar requirements to $\mathrm{FC}$, in terms of content availability, time to get content, content lifetime, success rate dependent on time spent inside the AZ [12], [32]. Starting from [12], [32], we will derive a set of engineering rules for FC in VANETs for the CONTACT architecture.

2) Mobility pattern-aware communications: Another special aspect of performance of the FC scheme, is its high sensitivity to the specific features of mobility patterns, to the 
replication strategy, and to the efficiency of the information exchange, e.g., time required to discover neighbors; time taken to set up/close a connection; effects of collisions and of interference. How to adaptively tune the main parameters of the floating content dependent on context, and in particular of adapting them to the characteristics of the mobility pattern, remains an open issue, particularly in vehicular environments. One of the special features of vehicle mobility patterns emerging from measurements is clustering, both in urban areas and on highways [35]. By allowing long lived exchanges of data between members, clusters clearly offer an opportunity for optimizing over vanilla FC. Through coordination mechanisms, nodes in a cluster could specialize and take up specific roles on behalf of other nodes in the cluster. For instance, techniques for decreasing FC traffic and reducing redundancy by means of proxying and caching could be devised. Some nodes could act as "storage nodes", keeping content alive and available to other cluster nodes, while others might specialize in coordinating communications and advertising the floating storage data objects. For instance, for enhancing inter-cluster communications, some nodes might act as cluster gateways.

Floating content schemes are typically implemented in a distributed fashion, without any orchestration or coordination. This is due to the opportunistic nature of the message exchanges, and more specifically, to the fact that the degree of connectivity of the resulting ad hoc network is very low, making it typically very hard to implement any form of decentralized orchestration and coordination. Nonetheless, we think that this is highly dependent on the specific setting (user density and speed, transmission range, and spatio-temporal patterns), and that in some specific (but not so rare) contexts (such as in vehicle clusters) some form of coordination schemes are feasible. Hence, an important aspect of our investigation will be the experimental assessment of those conditions in which some form of coordination between nodes participating in a FC scheme is feasible.

\section{CCN-SDN-FC INTEGRATION: PRELIMINARY IDEAS}

Adopting a cross-layer approach, we plan to integrate in a single architecture, (at least some of) the different techniques designed for optimizing $\mathrm{CCN}, \mathrm{SDN}$ and FC in VANETs, to the final aim of achieving high QoS support, by taking advantages of the benefits offered by each of them. In this Section we present some preliminary ideas on how to operate the integration of $\mathrm{CCN}, \mathrm{SDN}$ and $\mathrm{FC}$, in the CONTACT architecture.

\section{A. Orchestration and coordination}

In many practical settings, and in several of the futuristic ITS (intelligent transport systems) and smart city scenarios, indeed, fixed communication infrastructure is available, in the form of roadside units or cellular networks, for instance.
Cellular networks could be of interest for critical V2V communications, in the form of either direct or multi-hop M2M communications. In addition, SDN includes typically a coordination function, which could play a role in the management of FC message replication, and in the implementation of context aware caching strategies. Moreover, if we consider the user's smart-phone as an integrating part of a vehicle's communication system, we have a system capable of multihoming. In such scenarios, a specific mode of communication (e.g., cellular network technology) could be exploited for best effort, delay tolerant communications as well as for the exchange of control and coordination information.

More generally, we will explore how to take advantage of orchestration and coordination between nodes for FC operations. We will explore what kind of optimizations can be enabled by orchestration. This might bring to the formulation of a set of scheduling and optimization algorithms for a given cluster (or set of clusters) of vehicles.

\section{B. Content caching and forwarding}

CONTACT will consider the integration of the FC paradigm in the context of a content centric pull-based communication mode, in order to enhance content availability (i.e. in-network cache hits) in the network, as well as to minimize the probability of content disappearance due to network disconnection. In this case, the anchor zone (the geographical zone where the content is stored) moves with the vehicles. New models will have to be elaborated to evaluate the impact on the $\mathrm{CCN}$ routing techniques especially in terms of end to end delay and success rates in information retrieval. SDN-based methods for supporting forwarding, caching, and reducing replication (and consequently broadcasting), CCN-based VANETs will also be investigated within the project.

\section{CONCLUSIONS}

In this paper we have presented the approach taken in the CONTACT project to tackle the issue of designing a set of techniques and a general architecture for QoS provisioning in vehicular networks. The goal of the project is to investigate how an architecture for vehicular communications that integrates content-centric networking, software-defined networking as well as context aware floating content schemes can properly support the very diverse set of applications and services currently envisioned for vehicular environments. The improvements in communication reliability, content availability, and end-to-end delay will be pursued by adopting strategies based on the type of content (alerts, driving coordination, informational) as well as on its context attributes (such as location of origin, geographical range of interest, time of validity).

\section{ACKNOWLEDGEMENTS}

This publication was produced in the framework of the CONTACT project, funded by the Fonds National de la Recherche Luxembourg and the Swiss National Science Foundation (SNSF). 


\section{REFERENCES}

[1] S. Al-Sultan, M. M. Al-Doori, A. H. Al-Bayatti, and H. Zedan, "A comprehensive survey on vehicular ad hoc network," Journal of network and computer applications, vol. 37, pp. 380-392, 2014.

[2] T. L. Willke, P. Tientrakool, and N. F. Maxemchuk, "A survey of intervehicle communication protocols and their applications," Communications Surveys \& Tutorials, IEEE, vol. 11, no. 2, pp. 3-20, 2009.

[3] W. G. Najm, J. Koopmann, J. D. Smith, and J. Brewer, "Frequency of target crashes for intellidrive safety systems," Tech. Rep., 2010.

[4] E. Guizzo, "How googles self-driving car works," IEEE Spectrum Online, October, vol. 18, 2011.

[5] G. Motors, "GM EN-V," 2012. [Online]. Available: 'http://www.gm. com/vision/design_technology/emerging_technology.html'

[6] M. Fanaei, A. Tahmasbi-Sarvestani, Y. P. Fallah, G. Bansal, M. C. Valenti, and J. B. Kenney, "Adaptive content control for communication amongst cooperative automated vehicles," in Wireless Vehicular Communications (WiVeC), 2014 IEEE 6th International Symposium on. IEEE, 2014, pp. 1-7.

[7] S. Biswas, R. Tatchikou, and F. Dion, "Vehicle-to-vehicle wireless communication protocols for enhancing highway traffic safety," Communications Magazine, IEEE, vol. 44, no. 1, pp. 74-82, 2006.

[8] J. Peng and L. Cheng, "A distributed mac scheme for emergency message dissemination in vehicular ad hoc networks," Vehicular Technology, IEEE Transactions on, vol. 56, no. 6, pp. 3300-3308, 2007.

[9] S. Kumar, L. Shi, N. Ahmed, S. Gil, D. Katabi, and D. Rus, "Carspeak: a content-centric network for autonomous driving," ACM SIGCOMM Computer Communication Review, vol. 42, no. 4, pp. 259-270, 2012.

[10] V. Jacobson, D. K. Smetters, J. D. Thornton, M. F. Plass, N. H. Briggs, and R. L. Braynard, "Networking named content," in Proceedings of the 5th International Conference on Emerging Networking Experiments and Technologies, ser. CoNEXT '09. New York, NY, USA: ACM, 2009, pp. 1-12. [Online]. Available: http://doi.acm.org/10.1145/1658939.1658941

[11] ONF, "Software defined-networking: the new norm for networks," ONF white paper, 2012.

[12] A. A. V. Castro, G. D. M. Serugendo, and D. Konstantas, "Hovering information-self-organizing information that finds its own storage," in Autonomic Communication. Springer, 2009, pp. 111-145.

[13] E. Hyytiä, J. Virtamo, P. Lassila, J. Kangasharju, and J. Ott, "When does content float? characterizing availability of anchored information in opportunistic content sharing," in INFOCOM, 2011 Proceedings IEEE. IEEE, 2011, pp. 3137-3145.

[14] "Project CCNx," http://ccnx.org/, 2015, [Online; accessed 10-December2015].

[15] V. Jacobson, D. K. Smetters, N. H. Briggs, M. F. Plass, P. Stewart, J. D. Thornton, and R. L. Braynard, "Voccn: Voice-over content-centric networks," in Proceedings of the 2009 Workshop on Re-architecting the Internet, ser. ReArch '09. New York, NY, USA: ACM, 2009, pp. 1-6. [Online]. Available: http://doi.acm.org/10.1145/1658978.1658980

[16] G. Tyson, N. Sastry, I. Rimac, R. Cuevas, and A. Mauthe, "A survey of mobility in information-centric networks: Challenges and research directions," in Proceedings of the 1st ACM Workshop on Emerging Name-Oriented Mobile Networking Design - Architecture, Algorithms, and Applications, ser. NoM '12. New York, NY, USA: ACM, 2012, pp. 1-6. [Online]. Available: http://doi.acm.org/10.1145/2248361.2248363

[17] M. Varvello, I. Rimac, U. Lee, L. Greenwald, and V. Hilt, "On the design of content-centric manets," in Wireless On-Demand Network Systems and Services (WONS), 2011 Eighth International Conference on, Jan 2011, pp. 1-8.

[18] S. Oh, D. Lau, and M. Gerla, "Content centric networking in tactical and emergency manets," in Wireless Days (WD), 2010 IFIP, Oct 2010, pp. $1-5$.

[19] M. Meisel, V. Pappas, and L. Zhang, "Listen first, broadcast later: Topology-agnostic forwarding under high dynamics," Annual conference of international technology alliance in network and information science, p. $8,2010$.

[20] C. Anastasiades, T. Schmid, J. Weber, and T. Braun, "Opportunistic content-centric data transmission during short network contacts," in Wireless Communications and Networking Conference (WCNC), 2014 IEEE, April 2014, pp. 2516-2521.

[21] C. Anastasiades, T. Braun, and V. Siris, "Information-centric networking in mobile and opportunistic networks," in Wireless Networking for Moving Objects, ser. Lecture Notes in Computer Science, I. Ganchev, M. Curado, and A. Kassler, Eds. Springer International
Publishing, 2014, vol. 8611, pp. 14-30. [Online]. Available: http: //dx.doi.org/10.1007/978-3-319-10834-6_2

[22] L. Wang, A. Afanasyev, R. Kuntz, R. Vuyyuru, R. Wakikawa, and L. Zhang, "Rapid traffic information dissemination using named data," in Proceedings of the 1st ACM Workshop on Emerging Name-Oriented Mobile Networking Design - Architecture, Algorithms, and Applications, ser. NoM '12. New York, NY, USA: ACM, 2012, pp. 7-12. [Online]. Available: http://doi.acm.org/10.1145/2248361.2248365

[23] M. Amadeo, C. Campolo, A. Molinaro, and N. Mitton, "Named data networking: A natural design for data collection in wireless sensor networks," in Wireless Days (WD), 2013 IFIP, Nov 2013, pp. 1-6.

[24] C. Anastasiades and T. Braun, "Dynamic transmission modes to support opportunistic information-centric networks," in Networked Systems (NetSys), 2015 International Conference and Workshops on, March 2015, pp. $1-5$.

[25] Z. Zhao, T. Braun, D. Rosario, and E. Cerqueira, "Caor: Context-aware adaptive opportunistic routing in mobile ad-hoc networks," in Wireless and Mobile Networking Conference (WMNC), 2014 7th IFIP, May 2014, pp. 1-8.

[26] M. Yang, Y. Li, D. Jin, L. Zeng, X. Wu, and A. V. Vasilakos, "Softwaredefined and virtualized future mobile and wireless networks: a survey," Mobile Networks and Applications, vol. 20, no. 1, pp. 4-18, 2014.

[27] I. Ku, Y. Lu, M. Gerla, F. Ongaro, R. L. Gomes, and E. Cerqueira, "Towards software-defined vanet: Architecture and services," in Ad Hoc Networking Workshop (MED-HOC-NET), 2014 13th Annual Mediterranean. IEEE, 2014, pp. 103-110.

[28] N. B. Truong, G. M. Lee, and Y. Ghamri-Doudane, "Software defined networking-based vehicular adhoc network with fog computing," in Integrated Network Management (IM), 2015 Conference. IFIP/IEEE, 2015.

[29] K. Liu, J. K. Ng, V. Lee, S. H. Son, and I. Stojmenovic, "Cooperative data scheduling in hybrid vehicular ad hoc networks: Vanet as a software defined network," Transactions on Networking, IEEE/ACM, 2015.

[30] Y. Cao, J. Guo, and Y. Wu, "Sdn enabled content distribution in vehicular networks," in Innovative Computing Technology (INTECH), 2014 Fourth International Conference on. IEEE, 2014, pp. 164-169.

[31] Y.-C. Liu, C. Chen, and S. Chakraborty, "A software defined network architecture for geobroadcast in vanets," in Communications (ICC), 2015 IEEE International Conference on. IEEE, 2015, pp. 6559-6564.

[32] S. Ali, G. Rizzo, B. Rengarajan, and M. A. Marsan, "A simple approximate analysis of floating content for context-aware applications," in Proceedings of the fourteenth ACM international symposium on Mobile ad hoc networking and computing. ACM, 2013, pp. 271-276.

[33] B. Liu, B. Khorashadi, D. Ghosal, C.-N. Chuah, and M. H. Zhang, "Assessing the vanet's local information storage capability under different traffic mobility," in INFOCOM, 2010 Proceedings IEEE. IEEE, 2010, pp. $1-5$.

[34] I. Leontiadis, P. Costa, and C. Mascolo, "A hybrid approach for contentbased publish/subscribe in vehicular networks," Pervasive and Mobile Computing, vol. 5, no. 6, pp. 697-713, 2009.

[35] C. Shea, B. Hassanabadi, and S. Valaee, "Mobility-based clustering in vanets using affinity propagation," in Global Telecommunications Conference, 2009. GLOBECOM 2009. IEEE. IEEE, 2009, pp. 1-6. 